\title{
Engaging a wider audience
}

\author{
Nanoscience is not simple for non-specialists. Aravind Vijayaraghavan explains how graphene can help.
}

I $\mathrm{n}$ the last century, quantum physics has changed the way that we understand the universe, and by 'we', I of course mean scientists. For most of the nonscientific population, quantum physics remains a mystery. This is mainly due to the fact that we do not observe quantum phenomena in an obvious manner in our everyday life. The twenty-first century has heralded the age of nanoscience, dealing with structures so small that quantum phenomena manifest obviously. What's more, if scientific optimism is to be believed, we could be encountering these nanomaterials in everyday life sooner rather than later. There is, therefore, a pressing need for global awareness and indeed understanding of nanoscience.

\section{A new outreach tool}

Graphene offers an almost ideal tool to engage the non-scientific audience in nanoscience. The story of the 'origin' of graphene is fascinating and can captivate the imagination of even the youngest audience member. Two playful scientists one of whom had previously won the Ig-Nobel Prize for flying a frog - made a 'magic material' using pencil lead and sticky tape, and then went on to win the Nobel Prize and be knighted. Now everyone can get excited about a story like that. Even better, graphene isn't something too small to matter; under the right circumstances, a sheet of graphene 1 -atom thick is visible to the naked eye. One could even show off their institutional logo etched into a graphene sheet on a silicon substrate; that's the sort of thing that gets even marketing people in a university excited.

\section{My story}

Armed with a small optical microscope, some sticky tape, graphite powder and a giant prop pencil, I ventured to London in the summer of 2011 to exhibit at the Summer Science Exhibition. What I experienced there was truly inspirational. Thousands of people, from 8- to 80-year olds, queued for up to an hour, eager to have their bit of tape examined under the microscope to spot the graphene. The chocolate Nobel Prize medal for completing this task certainly helped add to the queue. The questions came thick and fast, from a child's simple curiosity to heated discussions with retired professors. The hands-on activities mostly stayed true to the original experiment, with a dose of excitement and creativity, but the activities all managed to get the core scientific message across without dumbing down the science. With a bit of training and patience, even first-year PhD students had no difficulty in explaining what a nanomaterial was to people irrespective of their scientific knowledge. The message I took home was that there was a deep and widespread appetite for nanoscience, and science in general. The crowds haven't got smaller at my graphene exhibits over the past 3 years, even though we ran out of the chocolate Nobel Prize medals a long time ago.

\section{Down from the ivory tower}

What surprised me almost as much was the level of enthusiasm among students, postdocs and even academics to step out of the lab and into the public square to communicate their breakthrough research in a down-to-earth manner. Most students who have done some science outreach will testify that explaining their project to a lay audience is not only a very rewarding feeling, but does wonders for their confidence. I'd strongly encourage anyone who hasn't yet tried it to give it a go; you'll be pleasantly surprised. To academic supervisors, all I can say is that it's OK to let your students out of the lab once in a while. For the particularly reluctant students, pick a music festival with a science pavilion. Even Glastonbury has one.

\section{A short rant}

My experience is in stark contrast to the constant recommendation that the best practice for scientists to attract the public's interest and attention, is to dumb down the science and exaggerate the potential of what we do, sometimes to ludicrous extents. People have a much greater hunger for science than they are given credit for. The media are perhaps the most to blame for this, with their constant efforts to appeal to the lowest common denominator. In the case of graphene, a statement that it is the thinnest, strongest, lightest material we have ever developed is very exciting in its own right, but correctly so, it doesn't have much relevance to the real world. One needs to put a statement like that in context. "So what?" is the question that always begs for an answer. The response that this could lead to components for the automotive and aerospace industry that are lighter and stronger is reasonable, but then it goes through the media spin cycle and emerges as 'electric aeroplanes made out of graphene within a few years'. In my experience, though, this is not necessary and is often detrimental.

\section{Dos and don'ts}

- Do have hands-on activities that can interest people of many age groups, such as molecular models.

- When a group/family visits your stand, do pay attention to the youngest member. If the child starts crying, you have lost your whole group.

- Do engage with your local science museum and science technology engineering maths (STEM) programmes for initial training in science communication and to reach our future scientists in local schools.

- Do share your amusing lab anecdotes, even at the risk of sound nerdy. Science jokes 'are' funny; the success of the TV series The Big Bang Theory is not a fluke.

- Offering a simple explanation or analogy is not the same as dumbing down the science.

- Don't sound patronising; it's better to assume people know more than less.

- Don't over-exaggerate the potential of what your research can accomplish.

- And finally, don't approach someone with "Hi, how're you doing?" or something similar. The instinctive response is always "Fine. (Now leave me alone)." Instead, try something more creative, such as my favourite opening "Would you like to make some graphene today?"

ARAVIND VIJAYARAGHAVAN is a lecturer and science communicator based in the School of Materials, University of Manchester, Oxford Road, Manchester M13 9PL, UK.

e-mail: aravind@manchester.ac.uk 